\title{
Relativistic redshift of the star S0-2 orbiting the Galactic center supermassive black hole
}

\author{
Tuan Do ${ }^{1 *}$, Aurelien Hees ${ }^{2,1}$, Andrea Ghez ${ }^{1}$, Gregory D. Martinez', Devin S. Chu', Siyao Jia ${ }^{3}$, Shoko Sakai', \\ Jessica R. Lu ${ }^{3}$, Abhimat K. Gautam', Kelly Kosmo O'Neil'1, Eric E. Becklin',4, Mark R. Morris', Keith Matthews ${ }^{5}$, \\ Shogo Nishiyama ${ }^{6}$, Randy Campbell ${ }^{7}$, Samantha Chappell ${ }^{1}$, Zhuo Chen ${ }^{1}$, Anna Ciurlo ${ }^{1}$, Arezu Dehghanfar ${ }^{1,8}$, \\ Eulalia Gallego-Cano ${ }^{9}$, Wolfgang E. Kerzendorf ${ }^{10,11,12,13}$, James E. Lyke ${ }^{7}$, Smadar Naoz ${ }^{1,14}$, Hiromi Saida ${ }^{15}$, Rainer \\ Schödel $^{9}$, Masaaki Takahashi ${ }^{16}$, Yohsuke Takamori ${ }^{17}$, Gunther Witzel ${ }^{1,18}$, Peter Wizinowich ${ }^{7}$
}

\begin{abstract}
${ }^{1}$ Department of Physics and Astronomy, University of California, Los Angeles, CA 90095, USA. 'Sytèmes de Référence Temps Espace, Observatoire de Paris, Université Paris-Sciences-et-Lettres, Centre national de la Recherche Scientifique, Sorbonne Université, Laboratoire National de métrologie et d'Essais, 61 avenue de l'Observatoire, 75014 Paris, France. ${ }^{3}$ Department of Astronomy, University of California, Berkeley, CA 94720-3411, USA. ${ }^{4}$ Universities Space Research Association/Stratospheric Observatory for Infrared Astronomy, NASA Ames Research Center, Mail Stop N232-12, Moffet Field, CA 94035, USA. 5 Division of Physics, Mathematics, and Astronomy, California Institute of Technology, MC 301-17, Pasadena, CA 91125, USA. ${ }^{\circ}$ Miyagi University of Education, 149 Aramaki-aza-aoba, Aoba-ku, Sendai, Miyagi 980-0845, Japan. ${ }^{7}$ W. M. Keck Observatory, 65-1120 Mamalahoa Highway, Kamuela, HI 96743, USA. ${ }^{8}$ Institut de Planétologie et d'Astrophysique de Grenoble, 414 Rue de la Piscine, 38400 Saint-Martin-d'Héres, France. ${ }^{9}$ Instituto de Astrofísica de Andalucía, Consejo Superior de Investigaciones Científicas, Glorieta de la Astronomía S/N, 18008 Granada, Spain. ${ }^{10}$ European Southern Observatory, Karl-Schwarzschild-Straß e 2,85748 Garching bei München, Germany. ${ }^{11}$ Center for Cosmology and Particle Physics, New York University, 726 Broadway, New York, NY 10003, USA. ${ }^{12}$ Department of Physics and Astronomy, Michigan State University, East Lansing, MI 48824, USA. ${ }^{13}$ Department of Computational Mathematics, Science, and Engineering, Michigan State University, East Lansing, MI 48824, USA. ${ }^{14}$ Mani L. Bhaumik Institute for Theoretical Physics, Department of Physics and Astronomy, University of California, Los Angeles, CA 90095, USA. ${ }^{15}$ Daido University, 10-3 Takiharu-cho, Minami-ku, Nagoya, Aichi 457-8530, Japan. ${ }^{16}$ Aichi University of Education, 1 Hirosawa, Igaya-cho, Kariya, Aichi 448-8542, Japan. ${ }^{17}$ National Institute of Technology, Wakayama College, 77 Noshima, Nada-cho, Gobo, Wakayama 644-0023, Japan. ${ }^{18}$ Max Planck Institute for Radio Astronomy, Auf dem Hügel 69, D-53121 Bonn (Endenich), Germany.

*Corresponding author. Email: tdo@astro.ucla.edu
\end{abstract}

General Relativity predicts that a star passing close to a supermassive black hole should exhibit a relativistic redshift. We test this using observations of the Galactic center star S0-2. We combine existing spectroscopic and astrometric measurements from 1995-2017, which cover S0-2's 16-year orbit, with measurements in 2018 March to September which cover three events during its closest approach to the black hole. We detect the combination of special relativistic- and gravitational-redshift, quantified using a redshift parameter, $\Upsilon$. Our result, $\Upsilon=0.88 \pm 0.17$, is consistent with General Relativity $(\Upsilon=1)$ and excludes a Newtonian model $(\Upsilon=0)$ with a statistical significance of $5 \sigma$.

General Relativity (GR) has been thoroughly tested in weak gravitational fields in the Solar System (1), with binary pulsars (2) and with measurements of gravitational waves from stellar-mass black-hole binaries $(3,4)$. Observations of shortperiod stars in our Galactic center (GC) (5-8) allow GR to be tested in a different regime (9): the strong field near a supermassive black hole (SMBH) $(10,11)$. The star S0-2 (also known as S2) has a 16 year orbit around Sagittarius $A^{*}\left(\operatorname{Sgr} A^{*}\right)$, the SMBH at the center of the Milky Way. In 2018 May, it reached its point of closest approach, at a distance of 120 astronomical units (au) with a velocity reaching $2.7 \%$ of the speed of light. Within a 6 months interval of that date, the star also passed through its maximum (March) and minimum velocity (September) along the line-of-sight, spanning a range of 6000 $\mathrm{km} \mathrm{s}^{-1}$ in radial velocity (RV - Fig. 1). We present observations of all three events and combine them with data from 19952017 (Fig. 2).

During 2018, the close proximity of S0-2 to the SMBH causes the relativistic redshift, which is the combination of the transverse Doppler shift from special relativity and the gravitational redshift from GR. This deviation from a Keplerian orbit was predicted to reach $200 \mathrm{~km} \mathrm{~s}^{-1}$ (Fig. 3) and is detectable with current telescopes. The GRAVITY collaboration (9) previously reported a similar measurement. Our measurements are complementary: i) we present a complete set of independent measurements with 3 additional months of data, doubling the time baseline for the year of closest approach, and including the third turning point (RV minimum) in September 2018, ii) we use three different spectroscopic instruments in 2018, which allows us to probe the presence of instrumental biases, iii) we perform an analysis of the systematic errors that may arise from an experiment spanning over 20 years to test for bias in the result, and iv) we publicly release the stellar measurements and the posterior probability distributions.

We use a total of 45 astrometric positional measurements (spanning 24 years) and $115 \mathrm{RVs}$ (18 years) to fit the orbit of S0-2. Of these, 11 are new astrometric measurements of S0-2 from 2016 to 2018 and 28 are new RV measurements from 2017 and 2018 (Fig. 1). Astrometric measurements were 
obtained at the W. M. Keck Observatory using speckle imaging (a technique to overcome blurring from the atmosphere by taking very short exposures and combining the images with software) from 1995-2005 and adaptive optics (AO) imaging (12) from 2005-2018. RV measurements were obtained from the W. M. Keck Observatory, Gemini North Telescope, and Subaru Telescope. All our RV observations were taken using AO. We supplement our observations with previously reported RVs from Keck from 2000 (7) and the Very Large Telescope (VLT) from 2003-2016 (8). This work includes data from a total of 2 imaging instruments and 6 spectroscopic instruments (13).

We scheduled our 2018 observations using a tool designed to maximize the sensitivity of the experiment to the redshift signal (13). Using this tool, we predicted that, given the existing data (1995-2017), spectroscopic measurements at the RV maximum and minimum in 2018 would provide the most sensitivity to detect the relativistic redshift (see Fig. 3). While they are less sensitive to the effect, imaging observations of the sky position of S0-2 in 2018 also slightly improve the measurement of the relativistic redshift.

The RVs of S0-2 are measured by fitting a physical model (which includes properties of the star such as its effective temperature, surface gravity, and rotational velocity in addition to RV), to its observed spectrum (13). The same procedure is applied to the new and archival observations; in the latter case this spectroscopic method improves the precision by a factor of 1.7 compared to previous analyses $(14,15)$.

We also characterized additional sources of uncertainties beyond the uncertainties in the fitted model: i) the wavelength solution, which transforms locations on the detector to vacuum wavelengths, was characterized by comparing the observed wavelengths of atmospheric $\mathrm{OH}$ emission lines in the spectra of S0-2 and in observations of blank sky to their known vacuum wavelengths. This comparison shows the uncertainty of the wavelength solution of the spectroscopic instruments to be about $2 \mathrm{~km} \mathrm{~s}^{-1}$, with some observations from 2002-2004 with lower accuracy between $2-26 \mathrm{~km} \mathrm{~s}^{-1}$. ii) Reexamination of the spectroscopic data showed that one spectroscopic instrument (NIRC2) had additional systematic bias from its optical system, which resulted in a systematic offset in RV compared to other instruments. We include an RV offset parameter in the orbit fit to account for this systematic offset. iii) We assessed systematic uncertainties by observations of bright RV standards stars of the same spectral-type as S0-2 (table S3). This systematic error is $1.3 \pm 1.2 \mathrm{~km} \mathrm{~s}^{-1}$, smaller than the statistical uncertainties and about 6 times smaller than previous RV observations of S0-2 (15). When these sources of systematic error are included in the analysis, the average RV uncertainty of S0-2 is found to be $20 \mathrm{~km} \mathrm{~s}^{-1}$ for the Keck and Gemini observations.

The astrometric positions of S0-2 with respect to Sgr A* are placed into a common absolute astrometric reference frame using a multi-step cross-matching and transformation process. We adopted an improved methodology for obtaining precise astrometry and a more accurate absolute reference frame compared to previous work (7). This resulted in an average astrometric uncertainty for S0-2 of 1.1 milliarcsecond (mas) for speckle imaging, and 0.26 mas for $\mathrm{AO}$ imaging.

The astrometric and RV measurements are combined in a global orbital model fitting using a standard post-Newtonian approximation which includes the first-order GR corrections on the Newtonian equations of motion, the Römer time delay due to variations in the light propagation time between S0-2 and the observer, and the relativistic redshift. For the astrometric observables, we ignore the negligible effect of light deflection by the SMBH but include a 2D linear drift of the gravitational center of mass. This drift accounts for systematic uncertainties in the construction of the astrometric reference frame. To our level of accuracy, the RV observable is (13)

$$
R V=v_{z_{0}}+V_{Z, \mathrm{~S} 0-2}+\Upsilon\left[\frac{V_{\mathrm{S} 0-2}^{2}}{2 c}+\frac{G M}{c R_{\mathrm{S} 0-2}}\right]
$$

where $c$ is the speed of light in a vacuum, $v_{z_{0}}$ is a constant offset introduced to account for systematic uncertainties within our RV reduction, $V_{Z, \mathrm{SO}-2}$ is the Newtonian line-ofsight velocity of S0-2, $V_{\mathrm{S} 0-2}^{2} / 2 c$ is the transverse Doppler shift predicted by special relativity depending on S0-2's velocity $\mathbf{V}_{\mathrm{S} 0-2}$ and $G M / c R_{\mathrm{S} 0-2}$ is the gravitational redshift predicted by GR incorporating the SMBH gravitational parameter $G M$ (the gravitational constant $G$, and SMBH mass $M$ ) and on the distance, $R_{\mathrm{S} 0-2}$, between S0-2 and the SMBH. $\Upsilon$ is a scale parameter introduced to characterize deviations from GR: its value is 0 in a purely Newtonian model and 1 in GR (13). The model has 14 parameters: 6 orbital parameters for S0-2, the gravitational parameter of the SMBH (GM), the distance to the Galactic center $R_{0}$, a 2-D linear drift of the SMBH parametrized by the 2-D position $\left(x_{0}, y_{0}\right)$ and velocity $\left(v_{x_{0}}, v_{y_{0}}\right)$ of the black hole from the center of the reference frame, an offset for the RV $v_{z_{0}}$, and the redshift parameter $\Upsilon$.

Several statistical tests were performed to assess systematic effects, using two different information criteria estimators to compare models: the Bayesian evidence and the expected logarithm predicted density (13). We examined several sources of systematic uncertainties in the orbital fit: (i) potential offsets in RVs and astrometric positions from different instruments and (ii) potentially correlated uncertainties in astrometric measurements. Based on Bayesian model selection, we find that one spectrograph requires a $\mathrm{RV}$ offset with respect to other instruments (likely due to optical 
fringing) (13). No other instruments require an RV or astrometric positional offset. We include a parameter for the spectrograph RV offset in the model so it is fitted simultaneously. Based on the model selection criteria, we also find spatial correlation in the astrometric uncertainties. The correlated uncertainties are modeled with a multivariate likelihood characterized by a covariance matrix. The correlation matrix introduces a characteristic correlation length scale $\lambda$ and a mixing parameter $p$, both of which are simultaneously fitted with the model parameters (13). We validated this approach by a Monte Carlo analysis, by randomly choosing one astrometric measurement per length scale to empirically estimate the effect of correlation scales. While the inclusion of these systematic effects does not significantly affect the best-fitting $\Upsilon$ value, it increases the uncertainties, affecting the precision of the results.

We developed an orbit modeling software package to model the orbits. The software uses Bayesian inference for model fitting, using nested sampling to estimate the posterior probability distribution via the multinest package $(16,17)$. We also perform Monte Carlo simulations to evaluate our fitting methodology and to show that the statistical uncertainties are robust (13).

We initially compare a purely Newtonian model with a purely relativistic ( $\Upsilon$ fixed to 1 ) model. We use the Bayes factor model selection criterion to show that the relativistic model is preferred by the data with high confidence. The difference of the logarithm of the Bayesian evidence between these two models is 10.68. Expressed as an odds ratio, the GR model is 43,000 times more likely than the Newtonian model in explaining the observations.

We then fitted the more general model that includes the $\Upsilon$ redshift parameter as a free parameter. The estimated values for the 17 fitted parameters are in Table 1 (the posterior distributions are shown in figs. S10 to S13). The estimation $\Upsilon=0.88 \pm 0.16$ and its marginal posterior is shown in Fig. 3C. We estimate the systematic uncertainties due to the astrometric reference frame construction by performing a jackknife analysis on stars used to construct the reference frame. This adds a systematic uncertainty on the redshift parameter of $\sim 0.047$, which when added in quadrature with the statistical uncertainties, results in a total uncertainty $\sigma_{\Upsilon}=0.17$. The measured redshift parameter is therefore $0.88 \pm 0.17$, consistent with GR at the $1 \sigma$ level while the Newtonian value $\Upsilon=0$ is excluded by $>5 \sigma$. Our estimation also agrees at the $1 \sigma$ level with the measurement by the GRAVITY collaboration (9). Our experiment is independent from theirs, using a different set of measurements that includes the third turning point. We examined additional sources of systematic error that were previously not considered. The best-fitting model to the RV and the fit residuals is presented in Fig. 2. A fit using a parameter encoding deviations from GR only at the level of the gravitational redshift gives $\alpha=-0.24 \pm 0.32$, where $\alpha=2(\Upsilon-1)$ is the standard gravitational redshift parameter $(1,13)$.

Our observations also constrain two other parameters: the mass of the black hole $\left(M_{\mathrm{BH}}\right)$ and the distance to the Galactic center $\left(R_{0}\right)$. From our model with $\Upsilon$ as free parameter, the $68 \%$ marginalized confidence interval for $M_{\mathrm{BH}}=(3.984 \pm$ $0.058 \pm 0.026) \times 10^{6} M_{\odot}$ and $R_{0}=7971 \pm 59 \pm 32 \mathrm{pc}$, where the first uncertainty is the statistical uncertainty and the second uncertainty is the systematic error $\sigma$ from the jackknife analysis (see Table 1). If we assume GR is true, then $M_{\mathrm{BH}}=$ $(3.964 \pm 0.047 \pm 0.026) \times 10^{6} M_{\odot}$ and $R_{0}=7946 \pm 50 \pm 32 \mathrm{pc}$ (see supplementary text for discussion). The nested sampling chains are provided in data S3.

The gravitational redshift is a direct consequence of the universality of free fall and of special relativity (18), hence of the Einstein equivalence principle, a fundamental principle of GR, which provides a geometric interpretation for gravitational interactions. Violations of the equivalence principle are predicted by some theories of modified gravity motivated by the development of a quantum theory of gravitation, unification theories, and some models of dark energy (19). While the gravitational redshift has been measured with higher precision within in the Solar System $(20,21)$, our results and those of the GRAVITY collaboration (9) extend the measurements to higher gravitational redshift and around a massive compact object, a SMBH. Sgr A* has a mass $\sim 4 \times 10^{6}$ times larger than that of the Sun. This constrains modified theories of gravitation that exhibit large non-perturbative effects around black holes, but not around non-compact objects like those in the Solar System (see (22-24) and supplementary text). This redshift test is also performed in a different environment than in the Solar System, where some theories predict modifications of GR to be screened or hidden (e.g., (25)).

\section{REFERENCES AND NOTES}

1. C. M. Will, The Confrontation between General Relativity and Experiment. Living Rev. Relativ. 17, 4 (2014). doi:10.12942//rr-2014-4 Medline

2. M. Kramer, Pulsars as probes of gravity and fundamental physics. Int. J. Mod. Phys. D 25, 1630029 (2016). doi:10.1142/S0218271816300299

3. B. P. Abbott et al.; LIGO Scientific and Virgo Collaborations, Tests of General Relativity with GW150914. Phys. Rev. Lett. 116, 221101 (2016). doi:10.1103/PhysRevLett.116.221101 Medline

4. B. P. Abbott et al., Gravitational Waves and Gamma-Rays from a Binary Neutron Star Merger: GW170817 and GRB 170817A. Astrophys. J. 848, L13 (2017). doi:10.3847/2041-8213/aa920c

5. R. Genzel, N. Thatte, A. Krabbe, H. Kroker, L. E. Tacconi-Garman, The Dark Mass Concentration in the Central Parsec of the Milky Way. Astrophys. J. 472, 153-172 (1996). doi:10.1086/178051

6. A. M. Ghez, B. L. Klein, M. Morris, E. E. Becklin, High Proper-Motion Stars in the Vicinity of Sagittarius A*: Evidence for a Supermassive Black Hole at the Center of Our Galaxy. Astrophys. J. 509, 678-686 (1998). doi:10.1086/306528

7. A. Boehle, A. M. Ghez, R. Schödel, L. Meyer, S. Yelda, S. Albers, G. D. Martinez, E. E. Becklin, T. Do, J. R. Lu, K. Matthews, M. R. Morris, B. Sitarski, G. Witzel, An improved distance and mass estimate for Sgr $A^{*}$ from a multistar orbit analysis. Astrophys. J. 830, 17 (2016). doi:10.3847/0004-637X/830/1/17 
8. S. Gillessen, P. M. Plewa, F. Eisenhauer, R. Sari, I. Waisberg, M. Habibi, O. Pfuhl, E. George, J. Dexter, S. Fellenberg, T. Ott, R. Genzel, An Update on Monitoring Stellar Orbits in the Galactic Center. Astrophys. J. 837, 30 (2017). doi:10.3847/15384357/aa5c41

9. Gravity Collaboration, R. Abuter, A. Amorim, N. Anugu, M. Bauböck, M. Benisty, J. P. Berger, N. Blind, H. Bonnet, W. Brandner, A. Buron, C. Collin, F. Chapron, Y. Clénet, V. C. du Foresto, P. T. de Zeeuw, C. Deen, F. Delplancke-Ströbele, R. Dembet, J. Dexter, G. Duvert, A. Eckart, F. Eisenhauer, G. Finger, N. M. F. Schreiber, P. Fédou, P. Garcia, R. G. Lopez, F. Gao, E. Gendron, R. Genzel, S. Gillessen, P. Gordo, M. Habibi, X. Haubois, M. Haug, F. Haußmann, T. Henning, S. Hippler, M. Horrobin, Z. Hubert, N. Hubin, A. J. Rosales, L. Jochum, L. Jocou, A. Kaufer, S. Kellner, S. Kendrew, P. Kervella, Y. Kok, M. Kulas, S. Lacour, V. Lapeyrère, B. Lazareff, J.-B. Le Bouquin, P. Léna, M. Lippa, R. Lenzen, A. Mérand, E. Müler, U. Neumann, T. Ott, L. Palanca, T. Paumard, L. Pasquini, K. Perraut, G. Perrin, 0. Pfuhl, P. M. Plewa, S. Rabien, A. Ramírez, J. Ramos, C. Rau, G. Rodríguez-Coira, R.-R. Rohloff, G. Rousset, J. Sanchez-Bermudez, S. Scheithauer, M. Schöller, N. Schuler, J. Spyromilio, O. Straub, C. Straubmeier, E. Sturm, L. J. Tacconi, K. R. W. Tristram, F. Vincent, S. von Fellenberg, I. Wank, I. Waisberg, F. Widmann, E. Wieprecht, M. Wiest, E. Wiezorrek, J. Woillez, S. Yazici, D. Ziegler, G. Zins, Detection of the gravitational redshift in the orbit of the star S2 near the Galactic centre massive black hole. Astron. Astrophys. 615, L15 (2018). doi:10.1051/0004-6361/201833718

10. D. Psaltis, X-ray Timing 2003: Rossi and Beyond, P. Kaaret, F. K. Lamb, J. H Swank, Eds. (2004), vol. 714 of American Institute of Physics Conference Series, pp. 29-35

11. T. Baker, D. Psaltis, C. Skordis, Linking tests of gravity on all scales: From the strong-field regime to cosmology. Astrophys. J. 802, 63 (2015). doi:10.1088/0004-637X/802/1/63

12. P. L. Wizinowich, D. Le Mignant, A. H. Bouchez, R. D. Campbell, J. C. Y. Chin, A. R. Contos, M. A. van Dam, S. K. Hartman, E. M. Johansson, R. E. Lafon, H. Lewis, P. J. Stomski, D. M. Summers, C. G. Brown, P. M. Danforth, C. E. Max, D. M. Pennington, The W. M. Keck Observatory Laser Guide Star Adaptive Optics System: Overview. Publ. Astron. Soc. Pac. 118, 297-309 (2006). doi:10.1086/499290

13. Materials and methods are available as supplementary materials.

14. A. M. Ghez, S. Salim, N. N. Weinberg, J. R. Lu, T. Do, J. K. Dunn, K. Matthews, M. R. Morris, S. Yelda, E. E. Becklin, T. Kremenek, M. Milosavljevic, J. Naiman, Measuring Distance and Properties of the Milky Way's Central Supermassive Black Hole with Stellar Orbits. Astrophys. J. 689, 1044-1062 (2008). doi:10.1086/592738

15. D. S. Chu, T. Do, A. Hees, A. Ghez, S. Naoz, G. Witzel, S. Sakai, S. Chappell, A. K. Gautam, J. R. Lu, K. Matthews, Investigating the Binarity of SO-2: Implications for Its Origins and Robustness as a Probe of the Laws of Gravity around a Supermassive Black Hole. Astrophys. J. 854, 12 (2018). doi:10.3847/15384357/aaa3eb

16. F. Feroz, M. P. Hobson, Multimodal nested sampling: An efficient and robust alternative to Markov Chain Monte Carlo methods for astronomical data analyses. Mon. Not. R. Astron. Soc. 384, 449-463 (2008). doi:10.1111/i.13652966.2007.12353.x

17. F. Feroz, M. P. Hobson, M. Bridges, MultiNest: An efficient and robust Bayesian inference tool for cosmology and particle physics. Mon. Not. R. Astron. Soc. 398 1601-1614 (2009). doi:10.1111/j.1365-2966.2009.14548.x

18. L. I. Schiff, On Experimental Tests of the General Theory of Relativity. Am. J. Phys. 28, 340-343 (1960). doi:10.1119/1.1935800

19. T. Damour, A. M. Polyakov, String theory and gravity. Gen. Relativ. Gravit. 26, 11711176 (1994). doi:10.1007/BF02106709

20. S. Herrmann, F. Finke, M. Lülf, O. Kichakova, D. Puetzfeld, D. Knickmann, M. List, B. Rievers, G. Giorgi, C. Günther, H. Dittus, R. Prieto-Cerdeira, F. Dilssner, F. Gonzalez, E. Schönemann, J. Ventura-Traveset, C. Lämmerzahl, Test of the Gravitational Redshift with Galileo Satellites in an Eccentric Orbit. Phys. Rev. Lett. 121, 231102 (2018). doi:10.1103/PhysRevLett.121.231102 Medline

21. P. Delva, N. Puchades, E. Schönemann, F. Dilssner, C. Courde, S. Bertone, F. Gonzalez, A. Hees, C. Le Poncin-Lafitte, F. Meynadier, R. Prieto-Cerdeira, B. Sohet, J. Ventura-Traveset, P. Wolf, Gravitational Redshift Test Using Eccentric Galileo Satellites. Phys. Rev. Lett. 121, 231101 (2018). doi:10.1103/PhysRevLett.121.231101 Medline

22. G. Antoniou, A. Bakopoulos, P. Kanti, Evasion of No-Hair Theorems and Novel
Black-Hole Solutions in Gauss-Bonnet Theories. Phys. Rev. Lett. 120, 131102 (2018). doi:10.1103/PhysRevLett.120.131102 Medline

23. H. O. Silva, J. Sakstein, L. Gualtieri, T. P. Sotiriou, E. Berti, Spontaneous Scalarization of Black Holes and Compact Stars from a Gauss-Bonnet Coupling. Phys. Rev. Lett. 120, 131104 (2018). doi:10.1103/PhysRevLett.120.131104 Medline

24. D. D. Doneva, S. S. Yazadjiev, New Gauss-Bonnet Black Holes with CurvatureInduced Scalarization in Extended Scalar-Tensor Theories. Phys. Rev. Lett. 120, 131103 (2018). doi:10.1103/PhysRevLett.120.131103 Medline

25. J. Khoury, A. Weltman, Chameleon fields: Awaiting surprises for tests of gravity in space. Phys. Rev. Lett. 93, 171104 (2004). doi:10.1103/PhysRevLett.93.171104 Medline

26. P. L. Wizinowich et al., Adaptive Optical Systems Technology, P. L. Wizinowich, Ed. (2000), vol. 4007 of Proc. SPIE, pp. 2-13.

27. A. M. Ghez, G. Duchêne, K. Matthews, S. D. Hornstein, A. Tanner, J. Larkin, M. Morris, E. E. Becklin, S. Salim, T. Kremenek, D. Thompson, B. T. Soifer, G. Neugebauer, I. McLean, The First Measurement of Spectral Lines in a Short-Period Star Bound to the Galaxy's Central Black Hole: A Paradox of Youth. Astrophys. J. 586, L127-L131 (2003). doi:10.1086/374804

28. J. Lyke, et al., OSIRIS Toolbox: OH-Suppressing InfraRed Imaging Spectrograph pipeline, Astrophysics Source Code Library, record ascl:1710.021 (2017).

29. M. A. van Dam, A. H. Bouchez, D. Le Mignant, E. M. Johansson, P. L. Wizinowich, R D. Campbell, J. C. Y. Chin, S. K. Hartman, R. E. Lafon, P. J. Stomski Jr., D. M. Summers, The W. M. Keck Observatory Laser Guide Star Adaptive Optics System: Performance Characterization. Publ. Astron. Soc. Pac. 118, 310-318 (2006). doi:10.1086/499498

30. T. Do, J. R. Lu, A. M. Ghez, M. R. Morris, S. Yelda, G. D. Martinez, S. A. Wright, K. Matthews, Stellar populations in the central $0.5 \mathrm{pc}$ of the Galaxy. I. A new method for constructing luminosity functions and surface-density profiles. Astrophys. J. 764, 154 (2013). doi:10.1088/0004-637X/764/2/154

31. G. Herriot et al., Adaptive Optical Systems Technology, P. L. Wizinowich, Ed. (2000), vol. 4007 of Proc. SPIE, pp. 115-125.

32. Nifty4gemini, https://github.com/mrlb05/nifty4gemini (2017).

33. M. Støstad, T. Do, N. Murray, J. R. Lu, S. Yelda, A. Ghez, Mapping the outer edge of the young stellar cluster in the Galactic center. Astrophys. J. 808, 106 (2015). doi:10.1088/0004-637X/808/2/106

34. A. Smette, H. Sana, S. Noll, H. Horst, W. Kausch, S. Kimeswenger, M. Barden, C. Szyszka, A. M. Jones, A. Gallenne, J. Vinther, P. Ballester, J. Taylor, Molecfit: A general tool for telluric absorption correction. Astron. Astrophys. 576, A77 (2015). doi:10.1051/0004-6361/201423932

35. N. Kobayashi et al., Optical and IR Telescope Instrumentation and Detectors, M. Iye, A. F. Moorwood, Eds. (2000), vol. 4008 of Proc. SPIE, pp. 1056-1066.

36. M. Iye, H. Karoji, H. Ando, N. Kaifu, K. Kodaira, K. Aoki, W. Aoki, Y. Chikada, Y. Doi, N. Ebizuka, B. Elms, G. Fujihara, H. Furusawa, T. Fuse, W. Gaessler, S. Harasawa, Y. Hayano, M. Hayashi, S. Hayashi, S. Ichikawa, M. Imanishi, C. Ishida, Y. Kamata, T. Kanzawa, N. Kashikawa, K. Kawabata, N. Kobayashi, Y. Komiyama, G. Kosugi, T. Kurakami, M. Letawsky, Y. Mikami, A. Miyashita, S. Miyazaki, Y. Mizumoto, J. Morino, K. Motohara, K. Murakawa, M. Nakagiri, K. Nakamura, H. Nakaya, K. Nariai, T. Nishimura, K. Noguchi, T. Noguchi, J. Noumaru, R. Ogasawara, N. Ohshima, Y. Ohyama, K. Okita, K. Omata, M. Otsubo, S. Oya, R. Potter, Y. Saito, T. Sasaki, S. Sato, D. Scarla, K. Schubert, K. Sekiguchi, M. Sekiguchi, I. Shelton, C. Simpson, H. Suto, A. Tajitsu, H. Takami, T. Takata, N. Takato, R. Tamae, M. Tamura, W. Tanaka, H. Terada, Y. Torii, F. Uraguchi, T. Usuda, M. Weber, T. Winegar, M. Yagi, T. Yamada, T. Yamashita, Y. Yamashita, N. Yasuda, M. Yoshida, M. Yutani, Current Performance and On-Going Improvements of the $8.2 \mathrm{~m}$ Subaru Telescope. Publ. Astron. Soc. Jpn. 56, 381-397 (2004). doi:10.1093/pasj/56.2.381

37. Y. Hayano et al., Adaptive Optics Systems II (2010), vol. 7736 of Proc. SPIE, p. $77360 \mathrm{~N}$.

38. S. Nishiyama, H. Saida, Y. Takamori, M. Takahashi, R. Schödel, F. Najarro, S. Hamano, M. Omiya, M. Tamura, M. Takahashi, H. Gorin, S. Nagatomo, T. Nagata, Radial velocity measurements of an orbiting star around Sgr A. Publ. Astron. Soc. Jpn. 70,74 (2018). doi:10.1093/pasj/psy067

39. R. C. Bohlin, S. Mészáros, S. W. Fleming, K. D. Gordon, A. M. Koekemoer, J. Kovács, A New Stellar Atmosphere Grid and Comparisons with HST /STIS CALSPEC Flux Distributions. Astron. J. 153, 234 (2017). doi:10.3847/1538-3881/aa6ba9 
40. M. Habibi, S. Gillessen, F. Martins, F. Eisenhauer, P. M. Plewa, O. Pfuhl, E. George, J. Dexter, I. Waisberg, T. Ott, S. Fellenberg, M. Bauböck, A. Jimenez-Rosales, R. Genzel, Twelve Years of Spectroscopic Monitoring in the Galactic Center: The Closest Look at S-stars near the Black Hole. Astrophys. J. 847, 120 (2017). doi:10.3847/1538-4357/aa876f

41. W. Kerzendorf, T. Do, Starkit: second release, 10.5281/zenodo.1117920 (2015).

42. T. Do, W. Kerzendorf, N. Winsor, M. Støstad, M. R. Morris, J. R. Lu, A. M. Ghez, Discovery of low-metallicity stars in the central parsec of the Milky Way. Astrophys. J. 809, 143 (2015). doi:10.1088/0004-637X/809/2/143

43. T. Do, W. Kerzendorf, Q. Konopacky, J. M. Marcinik, A. Ghez, J. R. Lu, M. R. Morris, Super-solar Metallicity Stars in the Galactic Center Nuclear Star Cluster: Unusual Sc, V, and Y Abundances. Astrophys. J. 855, L5 (2018). doi:10.3847/20418213/aaaec3

44. A. Feldmeier-Krause, W. Kerzendorf, N. Neumayer, R. Schödel, F. Nogueras-Lara, T. Do, P. T. de Zeeuw, H. Kuntschner, KMOS view of the Galactic Centre - II. Metallicity distribution of late-type stars. Mon. Not. R. Astron. Soc. 464, 194-209 (2017). doi:10.1093/mnras/stw2339

45. F. J. Kerr, D. Lynden-Bell, Review of galactic constants. Mon. Not. R. Astron. Soc. 221, 1023-1038 (1986). doi:10.1093/mnras/221.4.1023

46. S. Gillessen, F. Eisenhauer, S. Trippe, T. Alexander, R. Genzel, F. Martins, T. Ott, Monitoring stellar orbits around the massive black hole in the Galactic center. Astrophys. J. 692, 1075-1109 (2009). doi:10.1088/0004-637X/692/2/1075

47. G. A. Gontcharov, Pulkovo Compilation of Radial Velocities for 35495 Hipparcos stars in a common system. Astron. Lett. 32, 759-771 (2006). doi:10.1134/S1063773706110065

48. V. V. Bobylev, Open clusters IC 4665 and Cr 359 and a probable birthplace of the pulsar PSR B1929+10. Astron. Lett. 34, 686-698 (2008). doi:10.1134/S1063773708100046

49. W. Huang, D. R. Gies, M. V. McSwain, A stellar rotation census of B stars: From ZAMS to TAMS. Astrophys. J. 722, 605-619 (2010). doi:10.1088/0004$637 X / 722 / 1 / 605$

50. V. Khalack, F. LeBlanc, Project VeSElkA: Analysis of Balmer line profiles of slowly rotating chemically peculiar stars. Astron. J. 150, 2 (2015). doi:10.1088/0004$6256 / 150 / 1 / 2$

51. N. V. Kharchenko, R. D. Scholz, A. E. Piskunov, S. Röser, E. Schilbach, Astrophysical supplements to the ASCC-2.5: la. Radial velocities of $~ 55000$ stars and mean radial velocities of 516 Galactic open clusters and associations. Astron. Nachr. 328, 889-896 (2007). doi:10.1002/asna.200710776

52. T. Paumard, J.-P. Maillard, M. Morris, Kinematic and structural analysis of the Minispiral in the Galactic Center from BEAR spectro-imagery. Astron. Astrophys. 426, 81-96 (2004). doi:10.1051/0004-6361:20034209

53. S. Yelda, J. R. Lu, A. M. Ghez, W. Clarkson, J. Anderson, T. Do, K. Matthews, Improving Galactic center astrometry by reducing the effects of geometric distortion. Astrophys. J. 725, 331-352 (2010). doi:10.1088/0004$637 X / 725 / 1 / 331$

54. R. Schödel, S. Yelda, A. Ghez, J. H. Girard, L. Labadie, R. Rebolo, A. Pérez-Garrido, M. R. Morris, Holographic imaging of crowded fields: High angular resolution imaging with excellent quality at very low cost. Mon. Not. R. Astron. Soc. 429, 1367-1375 (2013). doi:10.1093/mnras/sts420

55. S. Jia, J. R. Lu, S. Sakai, A. K. Gautam, T. Do, M. W. Hosek Jr., M. Service, A. M. Ghez, E. Gallego-Cano, R. Schödel, A. Hees, M. R. Morris, E. Becklin, K. Matthews, The Galactic Center: Improved Relative Astrometry for Velocities, Accelerations, and Orbits near the Supermassive Black Hole. Astrophys. J. 873, 9 (2019). doi:10.3847/1538-4357/ab01de

56. S. Sakai, J. R. Lu, A. Ghez, S. Jia, T. Do, G. Witzel, A. K. Gautam, A. Hees, E. Becklin, K. Matthews, M. W. Hosek Jr., The Galactic Center: An Improved Astrometric Reference Frame for Stellar Orbits around the Supermassive Black Hole. Astrophys. J. 873, 65 (2019). doi:10.3847/1538-4357/ab0361

57. G. Witzel, G. Martinez, J. Hora, S. P. Willner, M. R. Morris, C. Gammie, E. E. Becklin, M. L. N. Ashby, F. Baganoff, S. Carey, T. Do, G. G. Fazio, A. Ghez, W. J. Glaccum, D. Haggard, R. Herrero-Illana, J. Ingalls, R. Narayan, H. A. Smith, Variability Timescale and Spectral Index of Sgr $A^{*}$ in the Near Infrared: Approximate Bayesian Computation Analysis of the Variability of the Closest Supermassive Black Hole. Astrophys. J. 863, 15 (2018). doi:10.3847/1538-4357/aace62

58. M. J. Reid, K. M. Menten, S. Trippe, T. Ott, R. Genzel, The Position of Sagittarius
A*. III. Motion of the Stellar Cusp. Astrophys. J. 659, 378-388 (2007). doi:10.1086/511744

59. C. M. Will, M. Maitra, Relativistic orbits around spinning supermassive black holes: Secular evolution to 4.5 post-Newtonian order. Phys. Rev. D 95, 064003 (2017). doi:10.1103/PhysRevD.95.064003

60. A. M. Ghez, S. Salim, S. D. Hornstein, A. Tanner, J. R. Lu, M. Morris, E. E. Becklin, G. Duchene, Stellar Orbits around the Galactic Center Black Hole. Astrophys. J. 620, 744-757 (2005). doi:10.1086/427175

61. V. A. Brumberg, Essential Relativistic Celestial Mechanics (Adam Hilger, 1991)

62. T. Damour, N. Deruelle, Ann. Inst. Henri Poincaré Phys. Théor. 44, 263 (1986).

63. A. Hees, S. Bertone, C. Le Poncin-Lafitte, Relativistic formulation of coordinate light time, Doppler, and astrometric observables up to the second postMinkowskian order. Phys. Rev. D 89, 064045 (2014). doi:10.1103/PhysRevD.89.064045

64. M. Grould, F. H. Vincent, T. Paumard, G. Perrin, General relativistic effects on the orbit of the S2 star with GRAVITY. Astron. Astrophys. 608, A60 (2017). doi:10.1051/0004-6361/201731148

65. L. Blanchet, C. Salomon, P. Teyssandier, P. Wolf, Relativistic theory for time and frequency transfer to order $c^{-3}$. Astron. Astrophys. 370, 320-329 (2001). doi:10.1051/0004-6361:20010233

66. T. Alexander, Stellar processes near the massive black hole in the Galactic center. Phys. Rep. 419, 65-142 (2005). doi:10.1016/j.physrep.2005.08.002

67. S. Zucker, T. Alexander, S. Gillessen, F. Eisenhauer, R. Genzel, Probing PostNewtonian Physics near the Galactic Black Hole with Stellar Redshift Measurements. Astrophys. J. 639, L21-L24 (2006). doi:10.1086/501436

68. W. M. Folkner, J. G. Williams, D. H. Boggs, R. S. Park, P. Kuchynka, Interplanetary Network Progress Report 196, 1 (2014)

69. B. Carpenter et al., J. Stat. Softw. Articles 76, 1 (2017).

70. L. Meyer, A. M. Ghez, R. Schödel, S. Yelda, A. Boehle, J. R. Lu, T. Do, M. R. Morris, E. E. Becklin, K. Matthews, The shortest-known-period star orbiting our Galaxy's supermassive black hole. Science 338, 84-87 (2012). doi:10.1126/science.1225506 Medline

71. A. Hees, T. Do, A. M. Ghez, G. D. Martinez, S. Naoz, E. E. Becklin, A. Boehle, S. Chappell, D. Chu, A. Dehghanfar, K. Kosmo, J. R. Lu, K. Matthews, M. R. Morris, S. Sakai, R. Schödel, G. Witzel, Testing General Relativity with Stellar Orbits around the Supermassive Black Hole in Our Galactic Center. Phys. Rev. Lett. 118, 211101 (2017). doi:10.1103/PhysRevLett.118.211101 Medline

72. B. J. Brewer, M. J. Francis, AIP Conference Proceedings (Springer, Berlin, Germany, 2009), vol. 1193, pp. 179-186.

73. E. T. Jaynes, Probability Theory: The Logic of Science (Cambridge University Press, Cambridge, 2003).

74. Y. Yao, A. Vehtari, D. Simpson, A. Gelman, arXiv:1704.02030 (2017).

75. R. E. Kass, A. E. Raftery, Bayes Factors. J. Am. Stat. Assoc. 90, 773-795 (1995). doi:10.1080/01621459.1995.10476572

76. H. Jeffreys, Theory of Probability (Oxford, Oxford, England, 1961), third edn.

77. H. Jeffreys, Some Tests of Significance, Treated by the Theory of Probability. Math. Proc. Camb. Philos. Soc. 31, 203-222 (1935). doi:10.1017/S030500410001330X

78. A. Gelman, J. Hwang, A. Vehtari, Understanding predictive information criteria for Bayesian models. Stat. Comput. 24, 997-1016 (2014). doi:10.1007/s11222-0139416-2

79. H. Akaike, (Budapest: Akademiai Kiado, 1973), pp. 267-281. Reprinted in Breakthroughs in Statistics, ed. S. Kotz, 610-624. New York: Springer (1992).

80. D. Spiegelhalter, N. Best, B. Carlin, A. van der Linde, Bayesian measures of model complexity and fit. J. R. Stat. Soc. B 64, 583-639 (2002). doi:10.1111/14679868.00353

81. S. Watanabe, arXiv:0712.0653 (2007).

82. S. Watanabe, arXiv:1004.2316 (2010).

83. A. Vehtari, A. Gelman, J. Gabry, Practical Bayesian model evaluation using leaveone-out cross-validation and WAIC. Stat. Comput. 27, 1413-1432 (2017). doi:10.1007/s11222-016-9696-4

84. L. B. Lucy, Mass estimates for visual binaries with incomplete orbits. Astron Astrophys. 563, A126 (2014). doi:10.1051/0004-6361/201322649

85. K. K. O'Neil, G. D. Martinez, A. Hees, A. M. Ghez, T. Do, G. Witzel, Q. Konopacky, E. E. Becklin, D. S. Chu, J. R. Lu, K. Matthews, S. Sakai, Improving Orbit Estimates for Incomplete Orbits with a New Approach to Priors: With Applications from Black 
Holes to Planets. Astron. J. 158, 4 (2019). doi:10.3847/1538-3881/ab1d66

86. J. Neyman, Outline of a Theory of Statistical Estimation Based on the Classical Theory of Probability. Philos. Trans. R. Soc. London Ser. A 236, 333-380 (1937). doi:10.1098/rsta.1937.0005

87. H. Rein, S.-F. Liu, REBOUND: An open-source multi-purpose $N$-body code for collisional dynamics. Astron. Astrophys. 537, A128 (2012). doi:10.1051/0004$6361 / 201118085$

88. A. Hees et al., arXiv:1809.05490 (2019).

89. T. Damour, J. F. Donoghue, Equivalence principle violations and couplings of a light dilaton. Phys. Rev. D 82, 084033 (2010). doi:10.1103/PhysRevD.82.084033

90. T. Damour, J. F. Donoghue, Phenomenology of the equivalence principle with light scalars. Class. Quantum Gravity 27, 202001 (2010). doi:10.1088/02649381/27/20/202001

91. T. Damour, Proceedings: 34th Rencontres de Moriond gravitational waves and experimental gravity: Les Arcs, France, Jan 23-30, 1999, J. Tran Thanh Van, et al., Eds. (Hanoi: The Gioi world Publishers, 1999), pp. 357-365.

92. A. Hees et al., arXiv:1906.03099 (2019).

\section{ACKNOWLEDGMENTS}

We thank the staff and astronomers at Keck Observatory and Gemini Observatory, especially Gary Puniwai, Jason Mcllroy, Sherry Yeh, John Pelletier, Joel Hicock, Greg Doppmann, Julie Renaud-Kim, Tony Ridenour, Alan Hatakeyama, Josh Walawender, Carolyn Jordan, Cynthia Wilburn, Terry Stickel, Heather Hershey, Jason Macilroy, John Pelletierm, Julie Renauld-Kim, Alessandro Rettura, Luca Rizzi, Carlos Alvarez, Marie Lemoine-Busserolle, Matthew Taylor, Trent Dupuy, Meg Schwamb, for all their help in obtaining the new data. The W.M. Keck Observatory is operated as a scientific partnership among the California Institute of Technology, the University of California, and the National Aeronautics and Space Administration. The authors wish to recognize that the summit of Maunakea has always held a very significant cultural role for the indigenous Hawaiian community. We are most fortunate to have the opportunity to observe from this mountain. We thank the Subaru telescope staff, especially Y. Minowa, T.-S. Pyo, J.-H. Kim, and E. Mieda, for their support for the Subaru observations. The Subaru Telescope is operated by the National Astronomical Observatory of Japan. Funding: Support for this work was provided by NSF AAG grant AST1412615, the W. M. Keck Foundation, the Heising-Simons Foundation, and the Gordon and Betty Moore Foundation. S.J. and J.R.L. acknowledge support from NSF AAG (AST-1518273). The W. M. Keck Observatory was made possible by the generous financial support of the W. M. Keck Foundation. S.N. acknowledges financial support by JSPS KAKENHI, Grant Number JP25707012, JP15K13463, JP18K18760, and JP19H00695. H.S. was supported by JSPS KAKENHI Grant Number JP26610050 and JP19H01900. Y.T. was supported by JSPS KAKENHI Grant Number JP26800150. M.T. was supported by JSPS KAKENHI Grant Number JP17K05439, and DAIKO FOUNDATION. W.E.K. was supported by an ESO Fellowship and the Excellence Cluster Universe, Technische Universitat München. R.S. and E.G. have received funding from the European Research Council under the European Union's Seventh Framework Programme (FP7/2007-2013)/ERC grant agreement no. [614922]. R.S. acknowledges financial support from the State Agency for Research of the Spanish MCIU through the "Center of Excellence Severo Ochoa" award for the Instituto de Astrofisica de Andalucia (SEV-2017-0709). Author contributions: A.M.G., T.D., J.R.L, M.R.M., E.E.B., K.M., A.H. contributed to conceptualization and design of the experiment. A.M.G., T.D., J.R.L., M.R.M., E.E.B., K.M., D.C., S.J., S.S., A.K.G., K.K.O., S.N., H.S., M.T., Y.T., R.C., Z.C., A.C., J.E.L., G.W., S.C. performed observations. T.D., D.C., S.N., S.C., A.C., participated in reducing spectroscopic data and making RV measurements. J.R.L., S.J., S.S., A.K.G., Z.C., G.W., R.S., E.G-O. reduced imaging data and made astrometric measurements. A.M.G., T.D., A.H., G.D.M., J.R.L., D.C., S.J., R.S., E.G-O., S.S., A.K.G., W.E.K., G.W., A.Z. participated in methodology development for improving astrometric and RV measurements. G.D.M., A.H., T.D. participated in statistical modeling and model comparisons. K.M., R.C., P.W., J.E.L. participated in building and improving instrumentation. All authors participated in writing and discussions of the paper. Competing interests: The authors declare no competing interests. Data and materials availability: Raw observational data are archived at: Keck Observatory https://www2.keck.hawaii.edu/koa/public/koa.php, Gemini
Observatory https://archive.gemini.edu/searchform, and Subaru https://smoka.nao.ac.jp/ under the dates and instruments listed in tables S1 and S4. Our reduced astrometric and RV measurements are provided in data S1 and S2. The nested sampling chains are provided in data S3. Software for the orbit modeling and scheduling tool are available at https://zenodo.org/record/3305315.

\section{SUPPLEMENTARY MATERIALS}

science.sciencemag.org/cgi/content/full/science.aav8137/DC1

Materials and Methods

Supplementary Text

Figs. S1 to S18

Tables S1 to S13

References (26-92)

Data S1 to S4

26 October 2018; accepted 11 July 2019

Published online 25 July 2019

10.1126/science.aav8137 


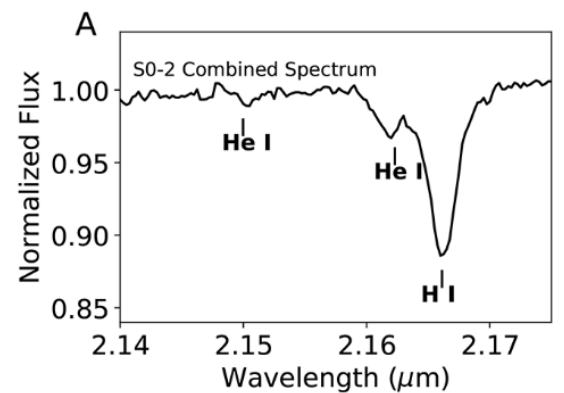

C

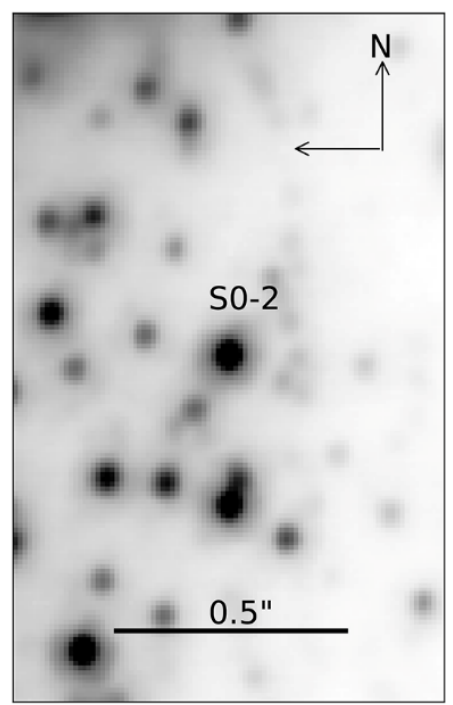

Keck $1.6 \mu \mathrm{m}$ image 2018 March

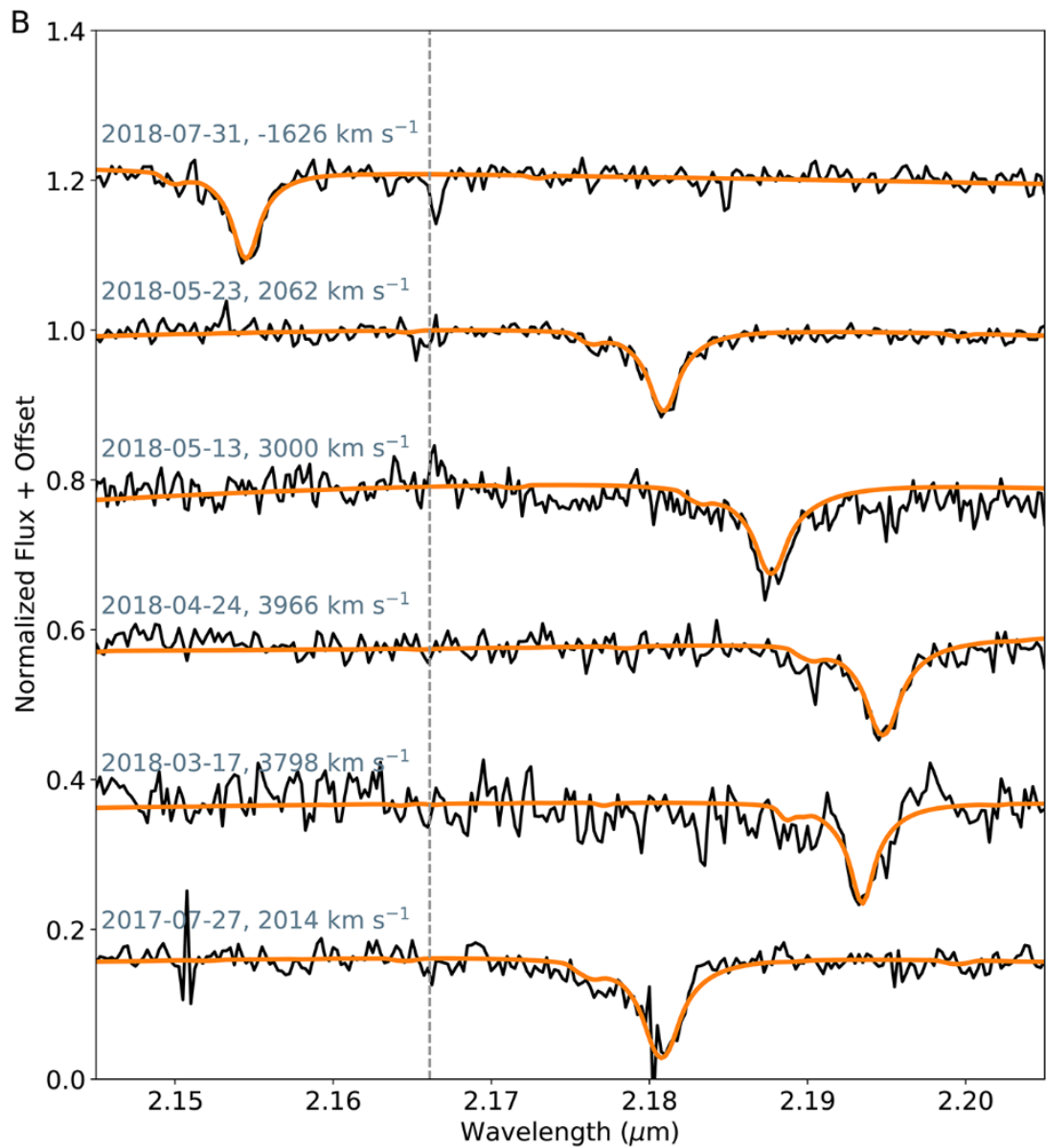

Fig. 1. Spectroscopy and imaging of the star S0-2. (A) A weighted-average spectrum of SO-2 from data obtained from 2006-2018 using Keck data. The strongest feature, which provides most of the RV constraint, is from the $\mathrm{H}$ i line at $2.1661 \mu \mathrm{m}$. (B) A sequence of SO-2 spectra observed in 2017 and 2018 (black lines). The RV of the star changes by over $6000 \mathrm{~km} \mathrm{~s}^{-1}$ throughout 2018 . The dashed line shows the rest wavelength of the $\mathrm{H}$ i line. We fit a model to the spectrum that simultaneously constrains the star's physical properties such as effective temperature and rotation along with the RV of the star (orange). This model accounts for the asymmetries in the $\mathrm{H}$ i feature. (C) An inverted Keck AO image of SO-2 (center of image) from March 2018 with the $\mathrm{H}$-band filter $(1.6 \mu \mathrm{m})$. 


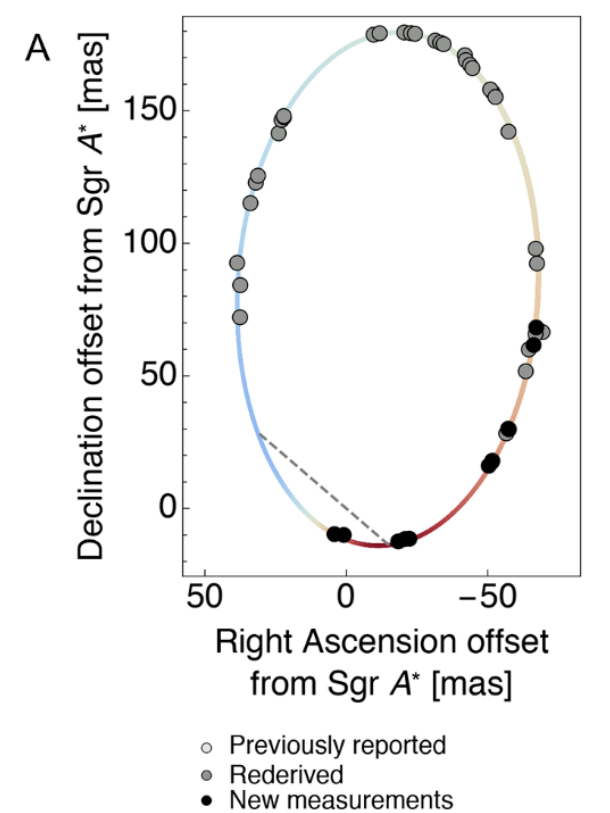

D क

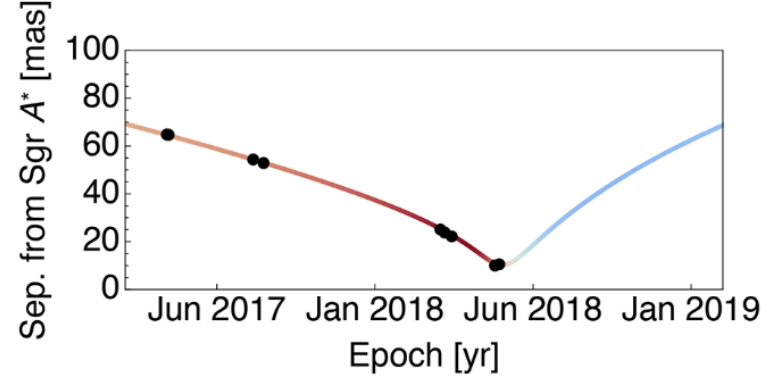

C
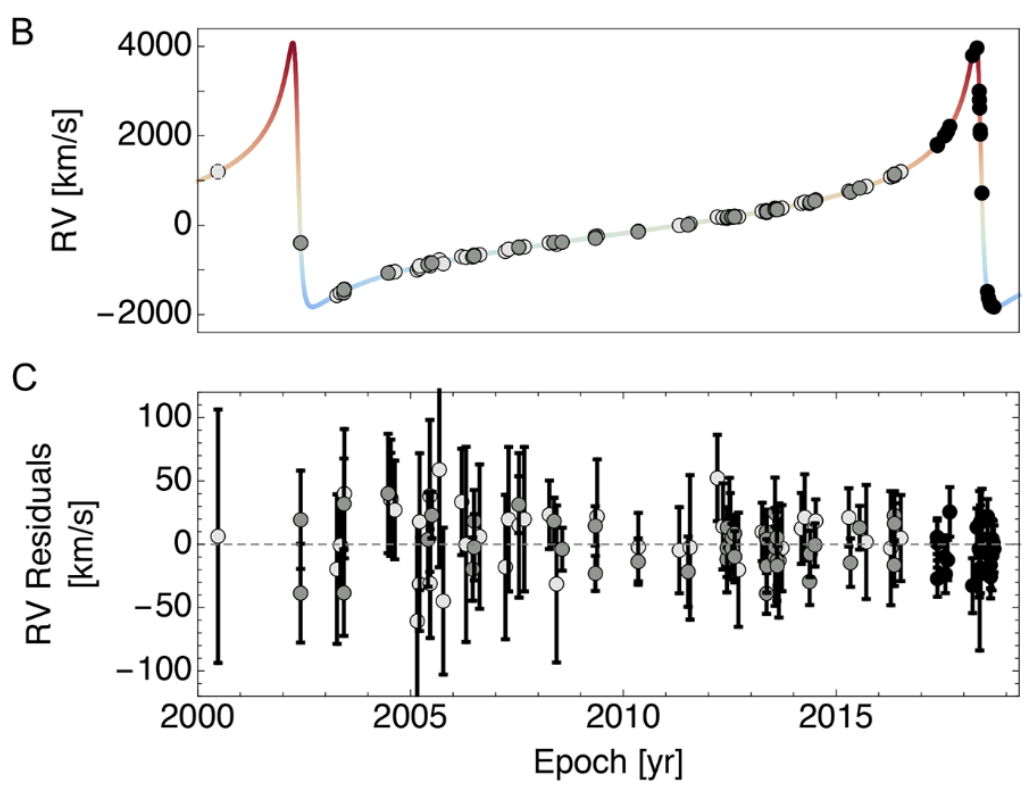

E

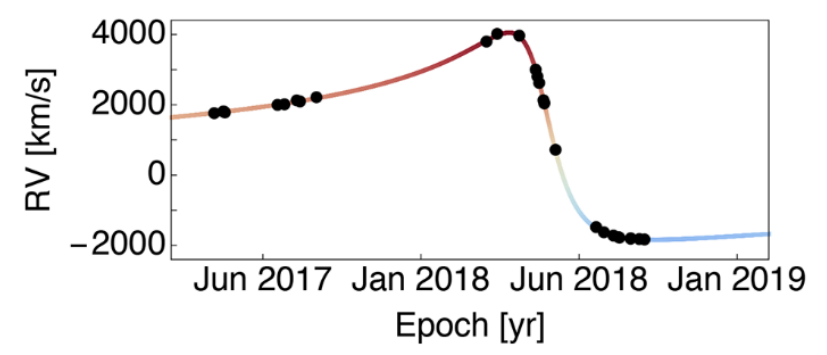

Fig. 2. GR orbit modeling of SO-2. (A) Astrometric measurements of the short-period star S0-2 in orbit around the SMBH (Sgr A*) overlain with our best-fitting projected orbit in the plane of the sky. The origin of the coordinate system coincides with the fitted SMBH center of mass (13). The $x$ and $y$ axes corresponds to offsets in right ascension and declination from the SMBH. 45 astrometric measurements from 1995-2018 of which 11 are new observations (black) and 34 rederived measurements (gray). The best-fitting SMBH linear drift has been removed from the measurements. The line of nodes (dashed line) shows the intersection of the orbital plane with the plane of the sky (this line also passes through the position of the black hole). S0-2 moves clockwise in this projection; the star is behind the black hole below the line of nodes and in front of the black hole above the line of nodes. The color and intensity used in the best-fitting orbital plot represent the direction and magnitude of the line-of-sight velocity with colors corresponding to panel (B). (B) RV measurements and the best-fitting RV model (colored line) using 115 RV measurements from 2000-2018. 42 measurements were previously reported (empty circles), 45 were rederived for this work with improved methodology (gray dots), and 28 are new observations (black dots). The color of the best-fitting orbit represents the value and sign of the line-of-sight velocity. (C) Residuals from the best-fitting RV model. ( $D$ and $E$ ) Observations around the three turning points, 1 at the closest approach to Sgr $A^{*}$ in the plane of the sky (D) and $2 \mathrm{RV}$ turning points [maximum and minimum RV, (E)] provide the greatest sensitivity to the relativistic redshift. 


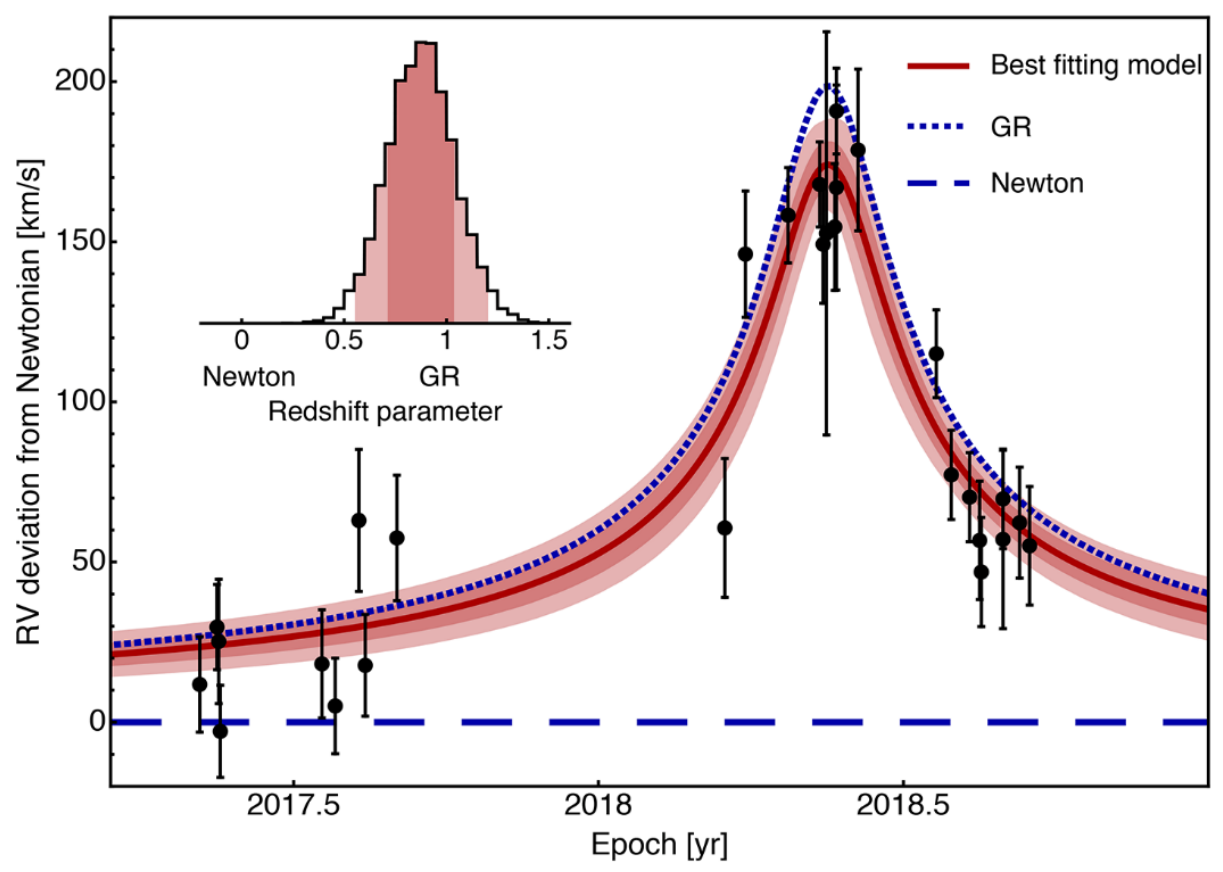

Fig. 3. Measured deviation from Newtonian predictions. The fitted deviation from Newtonian prediction, overlaid with the best-fitting orbit model (red line) corresponding to $\Upsilon=0.88$. The inset shows the posterior probability distribution for $\Upsilon ; 0.88$ is the median value. The red shaded areas show the model $68 \%$ and $95 \%$ confidence intervals. The observed $\mathrm{RV}$ s are shown as black points after removing the Newtonian part of the model. For comparison, we show the RV deviation expected for a purely relativistic signal ( $\Upsilon=1$, dotted blue line) and for a purely Newtonian model ( $\Upsilon=0$, dashed blue line) for an orbit with the same orbital parameters. Our measurement is consistent with the GR model at the $1 \sigma$ confidence level while the Newtonian model is excluded at $>5 \sigma$ confidence. 
Table 1. Estimation of the model parameters. Col 3: the maximum of the likelihood. Col 4: the median of the marginalized 1D posterior. Col 5: the half width of the $68 \%$ confidence interval centered around the median. Col 6 : the $1 \sigma$ systematics uncertainty from the reference frame estimated from the jackknife analysis (13).

\begin{tabular}{|c|c|c|c|c|c|}
\hline Parameter & Description & Max. likelihood & Estimation & $\begin{array}{c}\text { Statistical } \\
\text { uncertainty }\end{array}$ & $\begin{array}{c}\text { Systematic } \sigma \\
\text { from jackknife }\end{array}$ \\
\hline$M_{\mathrm{BH}}\left[10^{6} M_{\odot}\right]$ & Black hole mass & 3.984 & 3.975 & 0.058 & 0.026 \\
\hline$R_{0}[\mathrm{kpc}]$ & Distance to GC & 7.971 & 7.959 & 0.059 & 0.032 \\
\hline$\Upsilon<-3$ & Redshift parameter & 0.80 & 0.88 & 0.16 & 0.047 \\
\hline$x_{0}[\mathrm{mas}]$ & $x$ dynamical center & 0.99 & 1.22 & 0.32 & 0.51 \\
\hline$y_{0}[\mathrm{mas}]$ & $y$ dynamical center & -0.85 & -0.88 & 0.34 & 1.16 \\
\hline$v_{x_{0}}\left[\right.$ mas. $\left.\mathrm{yr}^{-1}\right]$ & $x$ velocity & -0.060 & -0.077 & 0.018 & 0.14 \\
\hline$v_{y_{0}}\left[\operatorname{mas} \mathrm{yr}^{-1}\right]$ & $y$ velocity & 0.221 & 0.226 & 0.019 & 0.066 \\
\hline$v_{z_{0}}[\mathrm{~km} / \mathrm{s}]$ & $z$ velocity & -3.6 & -6.2 & 3.7 & 0.79 \\
\hline$P[\mathrm{yr}]$ & Period & 16.041 & 16.042 & 0.0016 & $7.8 \times 10^{-5}$ \\
\hline$T_{0}[\mathrm{yr}]$ & Closest approach & 2018.3765 & 2018.3763 & 0.0004 & $1.9 \times 10^{-5}$ \\
\hline$e^{-l^{2}}$ & Eccentricity & 0.886 & 0.8858 & 0.0004 & $2.8 \times 10^{-5}$ \\
\hline$i$ [deg] & Inclination & 133.88 & 133.82 & 0.18 & 0.13 \\
\hline$\omega[\mathrm{deg}]$ & Argument of periapsis & 66.03 & 66.11 & 0.24 & 0.077 \\
\hline$\Omega[\mathrm{deg}]$ & Angle to the ascending node & 227.40 & 227.49 & 0.29 & 0.11 \\
\hline NIRC2 offset $\left[\mathrm{km} \cdot \mathrm{s}^{-1}\right]$ & RV offset & 80 & 81 & 19 & 0.8 \\
\hline$\Lambda$ [mas $]$ & Astrometric correlation length & 21 & 28 & $\begin{array}{r}24.6 \\
-13.6\end{array}$ & 11.8 \\
\hline$p$ & Astrometric mixing coefficient & 0.47 & 0.55 & 0.13 & 0.11 \\
\hline
\end{tabular}

